\title{
Laura skew group algebras
}

\author{
Ibrahim Assem*, Marcelo Lanzilotta ${ }^{\dagger}$ and María Julia Redondo ${ }^{\ddagger \S}$
}

\section{Introduction}

In the representation theory of artin algebras, an important line of research consists in studying those processes which allow to modify, in a predictable way, certain features of the module category of an artin algebra. In this paper, the features we are interested in are the left and the right parts of the module category, introduced by Happel, Reiten and Smalø in [12]. Let $A$ be an artin algebra, $\bmod A$ denote the category of finitely generated left $A$-modules and ind $A$ denote a full subcategory of $\bmod A$ having as objects exactly one representative from each isomorphism class of indecomposable $A$-modules. The left part $\mathcal{L}_{A}$ of $\bmod A$ is the full subcategory of ind $A$ having as objects those indecomposable $A$-modules whose predecessors have projective dimension at most one. The right part $\mathcal{R}_{A}$ is defined dually. These classes were used successfully in [12] to study the representation theory of quasi-tilted algebras, then, later, to study the many generalizations of this class such as the shod, weakly shod, laura and left (or right) supported algebras. However, the definition of $\mathcal{L}_{A}$ and $\mathcal{R}_{A}$ is not very practical: it is difficult to find all predecessors (or successors) of a given indecomposable module and thus to say whether it lies in $\mathcal{L}_{A}$ (or $\mathcal{R}_{A}$, respectively) or not. Our first theorem, which generalizes [12, II.1.5] and [9, 1.2], simplifies this task: it says that instead of considering all predecessors (or successors) of an indecomposable module, it suffices to look at the "immediate" ones.

THEOREM 1.1 Let $A$ be an artin algebra, and $M$ be an indecomposable A-module. Then:

(a) $M$ belongs to $\mathcal{L}_{A}$ if and only if, for every object $L$ in ind $A$ with projective dimension at least two, we have $\operatorname{Hom}_{A}(L, M)=0$.

(b) $M$ belongs to $\mathcal{R}_{A}$ if and only if, for every object $N$ in ind $A$ with injective dimension at least two, we have $\operatorname{Hom}_{A}(M, N)=0$.

As a first application of this theorem we consider the indecomposable Ext-injective modules in the additive subcategory of $\bmod A$ generated by $\mathcal{L}_{A}$, studied and characterized in $[3$, 3.1]. We give here handier characterizations.

\footnotetext{
*Département de Mathématiques, Université de Sherbrooke, Sherbrooke, Québec, Canada, J1K 2R1. Email address: ibrahim.assem@usherbrooke.ca

${ }^{\dagger}$ Centro de Matemática (CMAT), Iguá 4225, Universidad de la República, CP 11400, Montevideo, Uruguay.E-mail address: marclan@cmat.edu.uy

${ }^{\ddagger}$ Instituto de Matemática, Universidad Nacional del Sur, Av. Alem 1253, (8000) Bahía Blanca, Argentina. E-mail address: mredondo@criba.edu.ar

$\S^{\S}$ The third author is a researcher from CONICET, Argentina.
} 
Our main interest in this paper, however, lies in a construction which turns out to behave well when it comes to the left and the right parts: that of the skew group algebra. Let $G$ be a finite group acting on an artin algebra $A$, the skew group algebra $A[G]$ is the free left $A$-module with basis all the elements in $G$ endowed with the multiplication given by $(a \sigma)(b \zeta)=a \sigma(b) \sigma \zeta$ for all $a, b \in A$ and $\sigma, \zeta \in G$. The study of the representation theory of skew group algebras was started in $[16,13,14,15,11]$. We are partly motivated by the fact that finite coverings, as well as the smash algebras of [8], are particular cases of skew group algebras $[13,15,5]$. The algebra $A[G]$ retains many features from $A$ : indeed, let $G$ be a finite group whose order is invertible in $A$, then $A[G]$ is representation-finite (or an Auslander algebra, or a 1-Gorenstein algebra, or a self-injective algebra, or a Nakayama algebra) if and only if so is $A$ (see [16, (1.3)]). However, many properties are not preserved by this construction, like being a basic algebra, or being connected, so we are dealing with essentially different algebras, making it worthwhile to compare their representation theories.

We notice here that, since $A[G]$ is usually not basic, we need to consider instead a basic algebra $R=A[G]^{b}$ associated with $A[G]$. Clearly, such an algebra $R$ is only determined up to isomorphism, but this will pose no problem to us, since we are only interested in its module category, which is a Morita invariant. It is shown in [16] that the algebras $A$ and $R$ share most homological information. Thus, it has been shown that, if $A$ is a tilted (or a quasi-tilted) algebra, then so is $A[G]$, see [16] (or [12], respectively). However, for studying generalizations of these classes of algebras (such as, for instance, laura algebras) homological information, by itself, is not sufficient. We also need a nice correspondence between paths in ind $A$ and ind $R$. After establishing this correspondence, we are able to prove our second main theorem.

THEOREM 1.2 Let $A$ be an artin algebra, $G$ be a finite group acting on $A$ such that $|G|$ is invertible in $A$, and $R=A[G]^{b}$ be the basic algebra associated to the skew group algebra. Then:

(a) A is left (or right) supported if and only if so is $R$.

(b) $A$ is a laura algebra if and only if so is $R$.

(c) $A$ is left (or right) glued if and only if so is $R$.

(d) $A$ is weakly shod if and only if so is $R$.

(e) $A$ is shod if and only if so is $R$.

(f) $A$ is quasi-tilted if and only if so is $R$.

(g) $A$ is tilted if and only if so is $R$.

For the definitions of the above classes, we refer to $[1,2,3,9,10]$ or to Section 5 below. Here, statements (f) and (g) are included for completeness.

Finally, we apply this result to the toupie algebras of [7]. We define a new class, which we call skew toupie algebras, and exhibit a family of laura algebras (actually, weakly shod) skew toupie algebras. 


\section{A characterization of the left and right parts.}

\subsection{Notation.}

Let $A$ be an artin algebra. For a subcategory $\mathcal{C}$ of $\bmod A$, we write $M \in \mathcal{C}$ to express that $M$ is an object in $\mathcal{C}$. We denote by add $\mathcal{C}$ the full subcategory of $\bmod A$ having as objects the direct sums of indecomposable summands of objects in $\mathcal{C}$ and, if $M$ is an $A$-module, we abbreviate add $\{M\}$ as add $M$. We say that a full subcategory $\mathcal{C}$ of ind $A$ is finite if it has only finitely many objects. We denote the projective (or injective) dimension of an $A$-module $M$ by $\operatorname{pd}_{A} M$ (or $\operatorname{id}_{A} M$, respectively) and the global dimension of $A$ by gl.dim. $A$. Finally, we denote by $\Gamma(\bmod A)$ the Auslander-Reiten quiver of $A$, and by $\tau_{A}$ its Auslander-Reiten translation DTr. For further definitions or facts on $\bmod A, \Gamma(\bmod A)$, and $\tau_{A}$ we refer the reader to $[4,5]$.

Given $M, N \in$ ind $A$, we write $M \rightsquigarrow N$ in case there exists a path

$$
M=M_{0} \stackrel{f_{1}}{\rightarrow} M_{1} \rightarrow \cdots \rightarrow M_{t-1} \stackrel{f_{t}}{\rightarrow} M_{t}=N
$$

$(t \geq 0)$ from $M$ to $N$, that is, the $f_{i}$ are non-zero morphisms, and the $M_{i}$ are indecomposable $A$-modules. We then say that $M$ is a predecessor of $N$, and $N$ is a successor of $M$. If each $f_{i}$ in (1) is an irreducible morphism, we say that (1) is a path of irreducible morphisms. A path (1) of irreducible morphisms is sectional if $\tau_{A} M_{j+1} \neq M_{j-1}$ for all $j$ such that $1 \leq j \leq t$.

For the sake of brevity, from now on we refrain from stating the dual of each statement and leave the primal-dual translation to the reader.

The following result generalizes [12, II.1.5] and [9, 1.2] and its proof is inspired from the proofs of these statements (even though many details differ due to our more general hypothesis). Therefore, we give here, for the benefit of the reader, a complete proof.

LEMMA 2.1 Let $A$ be an artin algebra, and $M$ be an indecomposable $A$-module such that there exists a path $M_{0} \stackrel{f_{0}}{\rightarrow} M_{1} \stackrel{f_{1}}{\rightarrow} M$ in ind $A$ with $\operatorname{pd}_{A} M_{0} \geq 2$. Then there exists an indecomposable $A$-module $L$ with $\operatorname{pd}_{A} L \geq 2$ and $\operatorname{Hom}_{A}(L, M) \neq 0$.

Proof. Assume that this is not the case, that is, there exist $M \in \operatorname{ind} A$ and a path $M_{0} \stackrel{f_{0}}{\rightarrow} M_{1} \stackrel{f_{1}}{\rightarrow} M$ with $\operatorname{pd}_{A} M_{0} \geq 2$ and moreover $\operatorname{Hom}_{A}(L, M)=0$ for all $L \in$ ind $A$ with $\operatorname{pd}_{A} L \geq 2$. We may clearly choose the path $M_{0} \stackrel{f_{0}}{\rightarrow} M_{1} \stackrel{f_{1}}{\rightarrow} M$ with $\operatorname{pd}_{A} M_{0} \geq 2$ so that the length $\ell\left(M_{1}\right)$ of $M_{1}$ is minimal. It follows from our hypothesis that $f_{1} f_{0}=0$ (in particular, $f_{0}$ is not an epimorphism) and also that $\operatorname{pd}_{A} M_{1} \leq 1$.

We claim that $C=$ Coker $f_{0}$ is indecomposable. Since $f_{1} f_{0}=0$, we have $\operatorname{Hom}_{A}(C, M) \neq$ 0 . So, if $C$ were decomposable, there would exist an indecomposable direct summand $C^{\prime}$ of $C$ such that $\operatorname{Hom}_{A}\left(C^{\prime}, M\right) \neq 0$. Let $C \stackrel{p}{\rightarrow} C^{\prime} \stackrel{i}{\hookrightarrow} C$ denote respectively a projection and an injection such that $p i=1_{C^{\prime}}$. We have a fibered product diagram

$$
\begin{array}{ccccccccc}
0 & \rightarrow & \operatorname{Im} f_{0} & \stackrel{f^{\prime}}{\rightarrow} & M_{1}^{\prime} & \stackrel{g^{\prime}}{\rightarrow} & C^{\prime} & \rightarrow & 0 \\
& & & & \downarrow h & & \downarrow i & & \\
0 & \rightarrow & \operatorname{Im} f_{0} & \stackrel{f}{\rightarrow} & M_{1} & \stackrel{g}{\rightarrow} & C & \rightarrow & 0
\end{array}
$$


where $f$ is the canonical inclusion. We claim that the upper sequence is not zero. Indeed, assume that it is, and let $g^{\prime \prime}: C^{\prime} \rightarrow M_{1}^{\prime}$ be such that $g^{\prime} g^{\prime \prime}=1_{C^{\prime}}$. We have $(p g)\left(h g^{\prime \prime}\right)=$ $p(g h) g^{\prime \prime}=p\left(i g^{\prime}\right) g^{\prime \prime}=1_{C^{\prime}}$, hence $C^{\prime}$ is an indecomposable direct summand of $M_{1}$. Since $M_{1}$ is indecomposable, $C^{\prime} \simeq M_{1}$ and then $\operatorname{Im} f_{0}=0$, a contradiction. This shows that the upper sequence is not split.

Since, by hypothesis, $\operatorname{Hom}_{A}\left(C^{\prime}, M\right) \neq 0$ and $g^{\prime}$ is an epimorphism, $\operatorname{Hom}_{A}\left(M_{1}^{\prime}, M\right) \neq 0$. Thus, there exists an indecomposable direct summand $M_{1}^{\prime \prime}$ of $M_{1}^{\prime}$ such that $\operatorname{Hom}_{A}\left(M_{1}^{\prime \prime}, M\right) \neq$ 0 . On the other hand, $\operatorname{Im} f_{0}$ maps non-trivially on every indecomposable direct summand of $M_{1}^{\prime}$ (because the upper sequence is not split). In particular, $\operatorname{Hom}_{A}\left(\operatorname{Im} f_{0}, M_{1}^{\prime \prime}\right) \neq 0$. Composing with the canonical projection $f_{0}^{\prime}: M_{0} \rightarrow \operatorname{Im} f_{0}$ yields a nonzero morphism $M_{0} \rightarrow$ $M_{1}^{\prime \prime}$. Then we have a path $M_{0} \rightarrow M_{1}^{\prime \prime} \rightarrow M$ in ind $A$. Our minimality assumption yields $\ell\left(M_{1}\right) \leq \ell\left(M_{1}^{\prime \prime}\right) \leq \ell\left(M_{1}^{\prime}\right) \leq \ell\left(M_{1}\right)$ so that $M_{1} \simeq M_{1}^{\prime \prime}=M_{1}^{\prime}$. Therefore, $h$ is an isomorphism. Hence so is $i$. This establishes our claim that $C$ is indecomposable.

The indecomposability of $C$ implies that $\operatorname{pd}_{A} C \leq 1$, because $\operatorname{Hom}_{A}(C, M) \neq 0$. This, and the short exact sequence

$$
0 \rightarrow \operatorname{Im} f_{0} \stackrel{f}{\rightarrow} M_{1} \stackrel{g}{\rightarrow} C \rightarrow 0
$$

imply that $\operatorname{pd}_{A} \operatorname{Im} f_{0} \leq 1$. Therefore, $f_{0}$ is not a monomorphism.

On the other hand, $\operatorname{pd}_{A} C \leq 1$ implies $\operatorname{Ext}_{A}^{2}\left(C, \operatorname{Ker} f_{0}\right)=0$. In particular, the class in $\operatorname{Ext}_{A}^{2}\left(C, \operatorname{Ker} f_{0}\right)$ of the exact sequence

$$
0 \rightarrow \operatorname{Ker} f_{0} \rightarrow M_{0} \stackrel{f_{0}}{\rightarrow} M_{1} \stackrel{g}{\rightarrow} C \rightarrow 0
$$

vanishes. Letting $\epsilon_{1}$ and $\epsilon_{2}$ denote respectively the short exact sequences

$$
\left(\epsilon_{1}\right) \quad 0 \rightarrow \operatorname{Ker} f_{0} \rightarrow M_{0} \stackrel{f_{0}^{\prime}}{\rightarrow} \operatorname{Im} f_{0} \rightarrow 0, \quad\left(\epsilon_{2}\right) \quad 0 \rightarrow \operatorname{Im} f_{0} \stackrel{f}{\rightarrow} M_{1} \stackrel{g}{\rightarrow} C \rightarrow 0,
$$

we have $0=\epsilon=\epsilon_{1} \epsilon_{2}$. Applying $\operatorname{Hom}_{A}\left(-, \operatorname{Ker} f_{0}\right)$ to $\epsilon_{2}$ yields an exact sequence

$$
\cdots \rightarrow \operatorname{Ext}_{A}^{1}\left(M_{1}, \operatorname{Ker} f_{0}\right) \rightarrow \operatorname{Ext}_{A}^{1}\left(\operatorname{Im} f_{0}, \operatorname{Ker} f_{0}\right) \rightarrow \operatorname{Ext}_{A}^{2}\left(C, \operatorname{Ker} f_{0}\right)=0 .
$$

Then, there exists $\zeta \in \operatorname{Ext}_{A}^{1}\left(M_{1}, \operatorname{Ker} f_{0}\right)$ such that $\zeta f=\epsilon_{1}$. That is, there exists an $A$-module $N$ and a commutative diagram with exact rows and columns

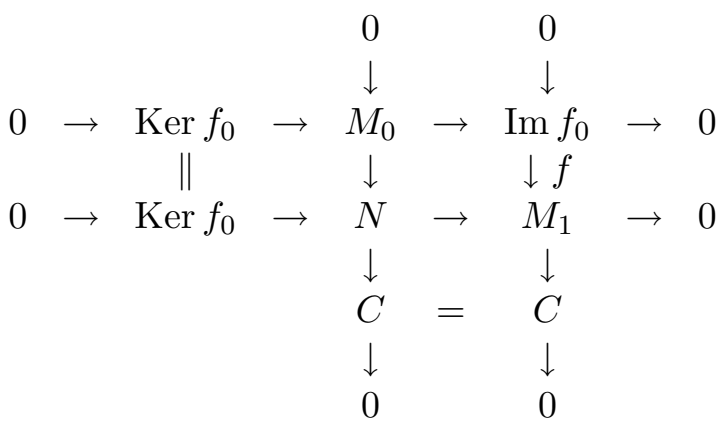


from which we deduce a short exact sequence

$$
0 \rightarrow M_{0} \rightarrow \operatorname{Im} f_{0} \oplus N \rightarrow M_{1} \rightarrow 0 .
$$

Since $\operatorname{pd}_{A} M_{0} \geq 2$ while $\operatorname{pd}_{A} \operatorname{Im} f_{0} \leq 1$ and $\operatorname{pd}_{A} M_{1} \leq 1$, then $N$ has an indecomposable summand $N^{\prime}$ with $\operatorname{pd}_{A} N^{\prime} \geq 2$. On the other hand, the middle column of the above diagram is not split (otherwise, the right column would split too, a contradiction). Hence, every indecomposable summand of $N$ maps non-trivially to $C$. We consider the resulting path $N^{\prime} \rightarrow C \rightarrow M$ in ind $A$. Since $\operatorname{pd}_{A} N^{\prime} \geq 2$ while $\ell(C)<\ell\left(M_{1}\right)$, we get a contradiction to our minimality assumption.

Proof of Theorem 1.1: It suffices to prove (a), since (b) is dual. Since the necessity of the condition is obvious, we prove the sufficiency. Assume that $M$ is such that, for every $L \in$ ind $A$ with $\operatorname{pd}_{A} L \geq 2$, we have $\operatorname{Hom}_{A}(L, M)=0$. We must show that every predecessor $L^{\prime}$ of $M$ has projective dimension at most one. Assume to the contrary that $\operatorname{pd}_{A} L^{\prime} \geq 2$, and use induction on the length $t$ of a shortest path from $L^{\prime}$ to $M$ in ind $A$ :

$$
L^{\prime}=M_{0} \stackrel{f_{1}}{\rightarrow} M_{1} \stackrel{f_{2}}{\rightarrow} M_{2} \ldots \stackrel{f_{t}}{\rightarrow} M_{t}=M .
$$

If $t \in\{0,1\}$, we have a contradiction to the hypothesis. If $t=2$, the contradiction follows from Lemma 2.1. Assume that $t \geq 3$. By Lemma 2.1, there exist an indecomposable $N$ and a non-zero morphism $f: N \rightarrow M_{2}$ such that $\operatorname{pd}_{A} N \geq 2$. But then the path

$$
N \stackrel{f}{\rightarrow} M_{2} \stackrel{f_{3}}{\rightarrow} M_{3} \ldots \stackrel{f_{t}}{\rightarrow} M_{t}=M
$$

of length $t-1$ yields a contradiction to the induction hypothesis.

\section{Ext-injectives in add $\mathcal{L}_{A}$.}

Let $A$ be a basic and connected artin algebra. We recall from [6] that an indecomposable module $M \in \mathcal{L}_{A}$ is called Ext-injective in add $\mathcal{L}_{A}$ whenever $\operatorname{Ext}_{A}^{1}(L, M)=0$ for all $L \in \mathcal{L}_{A}$. It is shown in $[6,3.4]$ that $M \in \mathcal{L}_{A}$ is Ext-injective in add $\mathcal{L}_{A}$ if and only if $\tau_{A}^{-1} M \notin \mathcal{L}_{A}$. Further, it is shown in $[3,3.1]$ that an indecomposable $A$-module $M$ is Ext-injective in add $\mathcal{L}_{A}$ if and only if it belongs to one of the following subsets of ind $A$ :

(a) $\mathcal{E}_{1}=\mathcal{E}_{1}(A)=\left\{L \in \mathcal{L}_{A}\right.$ : there exists an injective module $I \in$ ind $A$, and a path $I \rightsquigarrow L$ in ind $A\}$, and

(b) $\mathcal{E}_{2}=\mathcal{E}_{2}(A)=\left\{L \in \mathcal{L}_{A} \backslash \mathcal{E}_{1}\right.$ : there exists a projective module $P \in$ ind $A \backslash \mathcal{L}_{A}$, and a sectional path $\left.P \rightsquigarrow \tau_{A}^{-1} L\right\}$.

Clearly, $\mathcal{E}_{2}$ is contained in the (apparently) larger set:

$\mathcal{E}_{2}^{\prime}=\mathcal{E}_{2}^{\prime}(A)=\left\{L \in \mathcal{L}_{A} \backslash \mathcal{E}_{1}\right.$ : there exists a projective module $P \in$ ind $A \backslash \mathcal{L}_{A}$, and a path $\left.P \rightsquigarrow \tau_{A}^{-1} L\right\}$

We prove here that $\mathcal{E}_{2}=\mathcal{E}_{2}^{\prime}$. This yields an easier characterization of the Ext-injectives in add $\mathcal{L}_{A}$ which, apart from its theoretical interest, is used essentially in section 5 . 
PROPOSITION 3.1 Let $A$ be an artin algebra. An indecomposable A-module $M$ is Extinjective in add $\mathcal{L}_{A}$ if and only if $M \in \mathcal{E}_{1} \cup \mathcal{E}_{2}^{\prime}$.

Proof. Assume $M$ to be indecomposable and Ext-injective in add $\mathcal{L}_{A}$. Then $\tau_{A}^{-1} M \notin$ $\mathcal{L}_{A}$. By Theorem 1.1, there exists an indecomposable $A$-module $L$ such that $\operatorname{pd}_{A} L \geq 2$ and $\operatorname{Hom}_{A}\left(L, \tau_{A}^{-1} M\right) \neq 0$. Hence, there exists an indecomposable injective $I$ such that $\operatorname{Hom}_{A}\left(I, \tau_{A} L\right) \neq 0$. So, either $\operatorname{Hom}_{A}\left(\tau_{A} L, M\right) \neq 0$ and the path $I \rightarrow \tau_{A} L \rightarrow M$ yields $M \in \mathcal{E}_{1}$, or else $\operatorname{Hom}_{A}\left(\tau_{A} L, M\right)=0$ in which case the Auslander-Reiten formula gives $\underline{\operatorname{Hom}}_{A}\left(L, \tau_{A}^{-1} M\right) \simeq \overline{\operatorname{Hom}}_{A}\left(\tau_{A} L, M\right)=0$. Since $\operatorname{Hom}_{A}\left(L, \tau_{A}^{-1} M\right) \neq 0$, there exists an indecomposable projective module $P$ and a path $L \rightarrow P \rightarrow \tau_{A}^{-1} M$. Since $\operatorname{pd}_{A} L \geq 2$, we have $L \notin \mathcal{L}_{A}$, so $P \notin \mathcal{L}_{A}$ and consequently, $M \in \mathcal{E}_{2}^{\prime}$.

Conversely, let $M \in \mathcal{E}_{1} \cup \mathcal{E}_{2}^{\prime}$. Clearly, if $M \in \mathcal{E}_{2}^{\prime}$, then $M \in \mathcal{L}_{A}$ but $\tau_{A}^{-1} M \notin \mathcal{L}_{A}$ (because $\tau_{A}^{-1} M$ succedes a projective module not in $\mathcal{L}_{A}$ ) so that $M$ is Ext-injective in add $\mathcal{L}_{A}$. If $M \in \mathcal{E}_{1}$, then either $M$ is injective, or else there exists an indecomposable injective $I$ and a path $I \rightsquigarrow M \rightarrow * \rightarrow \tau_{A}^{-1} M$. By $[2,1.6]$, we infer that $\tau_{A}^{-1} M \notin \mathcal{L}_{A}$. Thus, $M$ is Ext-injective in add $\mathcal{L}_{A}$. This establishes the first assertion. The second follows immediately.

\section{Preliminaries on skew group algebras.}

We refer to $[5,16]$ for the basic definitions. Let $A$ be an artin $k$-algebra, and $G$ be a finite group with identity 1 acting on $A$. Such an action induces an action of $G$ on $\bmod A$ as follows. Let $M$ be an $A$-module, and $\sigma \in G$. We define ${ }^{\sigma} M$ to be the $A$-module with the additive structure of $M$ but where the multiplication is given by $a . x=\sigma^{-1}(a) x$, for $a \in A$ and $x \in M$.

LEMMA 4.1 Let $\sigma \in G$. The mapping $M \mapsto{ }^{\sigma} M$ (where $M$ is an A-module) induces an homomorphism of $G$ into the group of automorphisms of the category $\bmod A$.

Proof. We define a functor ${ }^{\sigma}(-): \bmod A \rightarrow \bmod A$ on objects by $M \mapsto{ }^{\sigma} M$. Let now $f: L \rightarrow M$ be a morphism of $A$-modules and define ${ }^{\sigma} f:{ }^{\sigma} L \rightarrow{ }^{\sigma} M$ by $x \mapsto f(x)$ for $x \in L$. This is an $A$-linear map because

$$
{ }^{\sigma} f(a . x)={ }^{\sigma} f\left(\sigma^{-1}(a) x\right)=f\left(\sigma^{-1}(a) x\right)=\sigma^{-1}(a) f(x)=a .{ }^{\sigma} f(x)
$$

for all $a \in A$ and $x \in L$. This clearly defines an endofunctor of $\bmod A$. The lemma then follows from the observation that, for any $\sigma_{1}, \sigma_{2} \in G$, we have ${ }^{\sigma_{1}}\left(\sigma_{2}(-)\right)=\sigma_{1} \sigma_{2}(-)$ and, in particular, ${ }^{\sigma}(-) .{ }^{-1}(-)=\operatorname{id}_{\bmod A}=\sigma^{-1}(-) .{ }^{\sigma}(-)$ for any $\sigma \in G$.

Assume that $G$ acts on $A$, and that the order $|G|$ of $G$ is invertible in $A$. Since the skew group algebra $A[G]$ is not basic in general, even if $A$ is so (see [5]), we agree to always consider the basic form $R=A[G]^{b}$ of $A[G]$. We stress that, as pointed out in the introduction, $R$ is only unique up to isomorphism. Also, in order to avoid confusion, we denote the $A$-modules by the letters $L, M, N, \ldots$ and the $R$-modules by the letters $X, Y, Z, \ldots$ 
The natural ring inclusion $A \hookrightarrow A[G]$ given by $a \mapsto a .1$ for $a \in A$, induces the change of rings functors $A[G] A[G]_{A} \otimes-: \bmod A \rightarrow \bmod A[G]$ and $\operatorname{Hom}_{A[G]}\left(A[G] A[G]_{A},-\right): \bmod A[G] \rightarrow$ $\bmod A$. Combining these functors with a (fixed) Morita equivalence $\bmod A[G] \simeq \bmod R$ yields functors $\bmod A \rightarrow \bmod R$ and $\bmod R \rightarrow \bmod A$, which we denote, by abuse of language, as $R \otimes_{A}-$ and $\operatorname{Hom}_{R}(R,-)$, respectively. The following proposition summarizes the properties of these functors, as stated and proved in [16, 1.1 and 1.8 (a)(b)(c)].

PROPOSITION 4.2 [Reiten-Riedtmann] Let $G$ be a finite group acting on an artin algebra $A$, and $R=A[G]^{b}$. Assume that $|G|$ is invertible in $A$. Then:

(a) $\left(R \otimes_{A}-, \operatorname{Hom}_{R}(R,-)\right)$ and $\left(\operatorname{Hom}_{R}(R,-), R \otimes_{A}-\right)$ are two adjoint pairs of functors.

(b) (1) The natural morphism $\operatorname{id}_{\bmod A} \rightarrow \operatorname{Hom}_{R}\left(R, R \otimes_{A}-\right)$ is a section of functors.

(2) The natural morphism $R \otimes_{A} \operatorname{Hom}_{R}(R,-) \rightarrow \mathrm{id}_{\bmod R}$ is a retraction of functors.

(c) If $M, N \in$ ind $A$, then

(1) $\operatorname{Hom}_{R}\left(R, R \otimes_{A} M\right) \simeq \bigoplus_{\sigma \in G}{ }^{\sigma} M$.

(2) $R \otimes_{A} M \simeq R \otimes_{A} N$ if and only if there exists $\sigma \in G$ such that $M \simeq{ }^{\sigma} N$.

(3) If $R \otimes_{A} M \simeq \bigoplus_{i=1}^{n} X_{i}$ is an indecomposable decomposition, then, for each $i$, the $A$-module $\operatorname{Hom}_{R}\left(R, X_{i}\right)$ has an indecomposable summand from each isomorphism class of the ${ }^{\sigma} M$, with $\sigma \in G$.

It follows from (a) above that both functors $R \otimes_{A}-$ and $\operatorname{Hom}_{R}(R,-)$ are exact and preserve projective and injective modules. On the other hand, as seen in (c), they do not preserve indecomposability.

COROLLARY 4.3 Let $X$ be an indecomposable $R$-module. Then there exists $M \in \operatorname{ind} A$ such that $M$ is a direct summand of $\operatorname{Hom}_{R}(R, X)$ and $X$ is a direct summand of $R \otimes_{A} M$.

Proof. By 4.2(b)(2), there is a retraction $R \otimes_{A} \operatorname{Hom}_{R}(R, X) \rightarrow X$. The statement follows from the indecomposability of $X$.

LEMMA 4.4 Let $M, N$ be indecomposable A-modules such that $\operatorname{Hom}_{A}(M, N) \neq 0$.

(a) For any indecomposable direct summand $X$ of $R \otimes_{A} M$, we have $\operatorname{Hom}_{R}\left(X, R \otimes_{A} N\right) \neq 0$.

(b) For any indecomposable direct summand $Y$ of $R \otimes_{A} N$, we have $\operatorname{Hom}_{R}\left(R \otimes_{A} M, Y\right) \neq 0$.

Proof. We only prove (a) since (b) is similar. By 4.2(c), we have an indecomposable decomposition in $\bmod R$

$$
R \otimes_{A} M=\oplus_{i=1}^{m} X_{i}
$$

such that $\operatorname{Hom}_{R}\left(R, X_{i}\right)=\bigoplus_{\sigma \in H_{i}}{ }^{\sigma} M$ for some $H_{i} \subset G$. Moreover, for any $i$, and any $\zeta \in G$, there exists $\sigma \in H_{i}$ such that ${ }^{\sigma} M \simeq \zeta M$. In particular, for any $i$ there exists $\sigma \in H_{i}$ such that ${ }^{\sigma} M \simeq M$. It follows from $\operatorname{Hom}_{A}(M, N) \neq 0$ that, for any $i, \operatorname{Hom}_{R}\left(X_{i}, R \otimes_{A} N\right) \simeq$ $\operatorname{Hom}_{A}\left(\operatorname{Hom}_{R}\left(R, X_{i}\right), N\right) \neq 0$. 
LEMMA 4.5 Let $M_{1} \rightarrow M_{2} \rightarrow \cdots \rightarrow M_{t}$ be a path in ind $A$.

(a) Then for any indecomposable direct summand $X_{1}$ of $R \otimes_{A} M_{1}$, there exists a path $X_{1} \rightarrow$ $X_{2} \rightarrow \cdots \rightarrow X_{t}$ in ind $R$ such that, for any $i, X_{i}$ is a direct summand of $R \otimes_{A} M_{i}$ and $M_{i}$ is a direct summand of $\operatorname{Hom}_{R}\left(R, X_{i}\right)$.

(b) For any indecomposable direct summand $Y_{t}$ of $R \otimes_{A} M_{t}$, there exists a path $Y_{1} \rightarrow Y_{2} \rightarrow$ $\cdots \rightarrow Y_{t}$ in ind $R$ such that, for any $i, Y_{i}$ is a direct summand of $R \otimes_{A} M_{i}$ and $M_{i}$ is a direct summand of $\operatorname{Hom}_{R}\left(R, Y_{i}\right)$.

Proof. We only prove (a) since (b) is similar. By 4.2(c)(3), since $X_{i}$ is a direct summand of $R \otimes_{A} M_{i}$, then $M_{i}$ is a direct summand of $\operatorname{Hom}_{R}\left(R, X_{i}\right)$ for any $i$. Since $X_{1}$ is a direct summand of $R \otimes_{A} M_{1}$ and $\operatorname{Hom}_{A}\left(M_{1}, M_{2}\right) \neq 0$, we have $\operatorname{Hom}_{A}\left(X_{1}, R \otimes_{A} M_{2}\right) \neq 0$ by 4.4(a). Hence there exists an indecomposable direct summand $X_{2}$ of the $R$-module $R \otimes_{A} M_{2}$ such that $\operatorname{Hom}_{R}\left(X_{1}, X_{2}\right) \neq 0$. The statement follows from an obvious induction.

LEMMA 4.6 Let $X, Y$ be indecomposable $R$-modules such that $\operatorname{Hom}_{R}(X, Y) \neq 0$. Let $M$ be an indecomposable summand of $\operatorname{Hom}_{R}(R, X)$ such that $X$ is a direct summand of $R \otimes_{A} M$. Then there exist $\sigma \in G$ and an indecomposable direct summand $N$ of $\operatorname{Hom}_{R}(R, Y)$ such that $Y$ is a direct summand of $R \otimes_{A}{ }^{\sigma} N$ and $\operatorname{Hom}_{A}\left(M,{ }^{\sigma} N\right) \neq 0$.

Proof. By 4.2(c)(2), $R \otimes_{A} N \simeq R \otimes_{A}{ }^{\sigma} N$ for all $\sigma \in G$ and all $N \in$ ind $A$. Since $X$ is a direct summand of $R \otimes_{A} M$ and $\operatorname{Hom}_{R}(X, Y) \neq 0$ then, for each $N \in$ ind $A$ such that $N$ is a direct summand of $\operatorname{Hom}_{R}(R, Y)$ and $Y$ is a direct summand of $R \otimes_{A} N$, we have $\operatorname{Hom}_{R}\left(R \otimes_{A} M, R \otimes_{A} N\right) \neq 0$. Adjunction gives $\operatorname{Hom}_{A}\left(M, \operatorname{Hom}_{R}\left(R, R \otimes_{A} N\right)\right) \neq$ 0 . By $4.2(\mathrm{c})(1), \operatorname{Hom}_{R}\left(R, R \otimes_{A} N\right) \simeq \bigoplus_{\sigma \in G}{ }^{\sigma} N$. Hence there exists $\sigma \in G$ such that $\operatorname{Hom}_{A}\left(M,{ }^{\sigma} N\right) \neq 0$ and $Y$ is a direct summand of $R \otimes_{A} N \simeq R \otimes_{A}{ }^{\sigma} N$.

LEMMA 4.7 Let $X_{1} \rightarrow X_{2} \rightarrow \cdots \rightarrow X_{t}$ be a path in ind $R$.

(a) Then for any indecomposable direct summand $M_{1}$ of $\operatorname{Hom}_{R}\left(R, X_{1}\right)$ such that $X_{1}$ is a direct summand of $R \otimes_{A} M_{1}$, there exist $\sigma_{2}, \cdots, \sigma_{t} \in G$ and a path

$$
M_{1} \rightarrow{ }^{\sigma_{2}} M_{2} \rightarrow \cdots \rightarrow{ }^{\sigma_{t-1}} M_{t-1} \rightarrow{ }^{\sigma_{t}} M_{t}
$$

in ind $A$, with $M_{i}$ a direct summand of $\operatorname{Hom}_{R}\left(R, X_{i}\right)$ and $X_{i}$ a direct summand of $R \otimes_{A}{ }^{\sigma_{i}} M_{i}$ for any $i$.

(b) For any indecomposable direct summand $N_{t}$ of $\operatorname{Hom}_{R}\left(R, X_{t}\right)$ such that $X_{t}$ is a direct summand of $R \otimes_{A} N_{t}$, there exist $\zeta_{1}, \cdots, \zeta_{t-1} \in G$ and a path

$$
{ }^{\zeta_{1}} N_{1} \rightarrow^{\zeta_{2}} N_{2} \rightarrow \cdots \rightarrow{ }^{\zeta_{t-1}} N_{t-1} \rightarrow N_{t}
$$

in ind $A$, with $N_{i}$ a direct summand of $\operatorname{Hom}_{R}\left(R, X_{i}\right)$ and $X_{i}$ a direct summand of $R \otimes_{A}^{\zeta_{i}} N_{i}$ for any $i$.

Proof. The proof is easy and left to the reader. 


\section{Laura skew group algebras.}

Throughout this section, we assume that $A$ is a basic and connected artin algebra, that $G$ is a group acting on $A$, with $|G|$ invertible in $A$, and we let $R=A[G]^{b}$.

LEMMA 5.1 For any $\sigma \in G$, we have ${ }^{\sigma} \mathcal{L}_{A}=\mathcal{L}_{A}$.

Proof. We first show that ${ }^{\sigma} \mathcal{L}_{A} \subseteq \mathcal{L}_{A}$. Let $L \in{ }^{\sigma} \mathcal{L}_{A}$, then ${ }^{\sigma-1} L \in \mathcal{L}_{A}$. Let $M$ be an indecomposable $A$-module such that $\operatorname{Hom}_{A}(M, L) \neq 0$. Then $\operatorname{Hom}_{A}\left({ }^{\sigma^{-1}} M, \sigma^{-1} L\right) \neq 0$. Since ${ }^{\sigma^{-1}} L \in \mathcal{L}_{A}$, we have $\operatorname{pd}_{A}{ }^{\sigma^{-1}} M \leq 1$. Hence $\operatorname{pd}_{A} M \leq 1$. By Theorem 1.1, $L \in \mathcal{L}_{A}$, as required. Conversely, if $N \in \mathcal{L}_{A}$, then ${ }^{\sigma^{-1}} N \in{ }^{\sigma^{-1}} \mathcal{L}_{A}$ so that ${ }^{\sigma^{-1}} N \in \mathcal{L}_{A}$ and $N \in{ }^{\sigma} \mathcal{L}_{A}$.

LEMMA $5.2 \quad$ (a) $\operatorname{add}\left(R \otimes_{A} \mathcal{L}_{A}\right)=\operatorname{add} \mathcal{L}_{R}$.

(b) $\operatorname{add} \operatorname{Hom}_{R}\left(R, \mathcal{L}_{R}\right)=\operatorname{add} \mathcal{L}_{A}$.

Proof. We first show that $\operatorname{add}\left(R \otimes_{A} \mathcal{L}_{A}\right) \subseteq \operatorname{add} \mathcal{L}_{R}$. Let $M \in \mathcal{L}_{A}$, and $R \otimes_{A} M=\bigoplus_{i=1}^{m} X_{i}$ be a decomposition into indecomposable modules in $\bmod R$. We claim that $X_{i} \in \mathcal{L}_{R}$ for any $i$. By Theorem 1.1, we must prove that, for each $Y \in$ ind $R$ such that $\operatorname{Hom}_{R}\left(Y, X_{i}\right) \neq 0$, we have $\operatorname{pd}_{R} Y \leq 1$. By 4.6, there exist $L, N \in$ ind $A$ such that $L$ is a direct summand of $\operatorname{Hom}_{R}(R, Y), Y$ is a direct summand of $R \otimes_{A} L, N$ is a direct summand of $\operatorname{Hom}_{R}\left(R, X_{i}\right)$, $X_{i}$ is a direct summand of $R \otimes_{A}{ }^{\sigma} N$ for some $\sigma \in G$, and $\operatorname{Hom}_{A}\left(L,{ }^{\sigma} N\right) \neq 0$. On the other hand, by $4.2(\mathrm{c})$, we have an indecomposable decomposition in $\bmod A$

$$
\operatorname{Hom}_{R}\left(R, X_{i}\right)=\oplus_{\zeta \in H_{i}}{ }^{\zeta} M
$$

for some $H_{i} \subseteq G$. Hence, there exists $\zeta \in H_{i}$ such that $\operatorname{Hom}_{A}\left(L,{ }^{\sigma \zeta} M\right) \neq 0$. By 5.1, ${ }^{\sigma \zeta} M \in \mathcal{L}_{A}$ hence $\operatorname{pd}_{A} L \leq 1$. Therefore $\operatorname{pd}_{R} R \otimes_{A} L \leq 1$. Since $Y$ is a direct summand of $R \otimes_{A} L$, we infer that $\operatorname{pd}_{R} Y \leq 1$, as required.

We next prove that add $\operatorname{Hom}_{R}\left(R, \mathcal{L}_{R}\right) \subseteq \operatorname{add} \mathcal{L}_{A}$. Let $X \in \mathcal{L}_{R}$ and $\operatorname{Hom}_{R}(R, X)=$ $\oplus_{i=1}^{m} M_{i}$ be a decomposition into indecomposable modules in $\bmod A$. We claim that $M_{i} \in \mathcal{L}_{A}$ for any $i$. By Theorem 1.1, we must prove that for each $L \in$ ind $A$ such that $\operatorname{Hom}_{A}\left(L, M_{i}\right) \neq$ 0 , we have $\operatorname{pd}_{A} L \leq 1$. Let $R \otimes_{A} L=\bigoplus_{j=1}^{n} Y_{j}$ be an indecomposable decomposition in $\bmod R$. Since $\operatorname{Hom}_{A}\left(L, M_{i}\right) \neq 0$, we have $\operatorname{Hom}_{R}\left(R \otimes_{A} L, X\right) \simeq \operatorname{Hom}_{A}\left(L, \operatorname{Hom}_{R}(R, X)\right) \neq 0$, so there exists $j_{0} \in\{1, \cdots, n\}$ such that $\operatorname{Hom}_{R}\left(Y_{j_{0}}, X\right) \neq 0$. Then $\operatorname{pd}_{R} Y_{j_{0}} \leq 1$. On the other hand, $\operatorname{Hom}_{R}\left(R, Y_{j_{0}}\right) \simeq \bigoplus_{\sigma \in H_{j_{0}}}{ }^{\sigma} L$ for some $H_{j_{0}} \subseteq G$. Hence $\operatorname{pd}_{R} Y_{j_{0}}=\operatorname{pd}_{A} \operatorname{Hom}_{R}\left(R, Y_{j_{0}}\right)=$ $\operatorname{pd}_{A}{ }^{\sigma} L=\operatorname{pd}_{A} L$ for each $\sigma \in H_{j_{0}}$. Therefore, $\operatorname{pd}_{A} L \leq 1$.

There remains to prove that equality holds in each case. Assume $X \in \mathcal{L}_{R}$. Then $\operatorname{Hom}_{R}(R, X) \in \operatorname{add} \mathcal{L}_{A}$. By $4.2(\mathrm{~b}), X$ is a direct summand of $R \otimes_{A} \operatorname{Hom}_{R}(R, X)$. Hence $X \in \operatorname{add}\left(R \otimes_{A} \mathcal{L}_{A}\right)$. Similarly, if $M \in \mathcal{L}_{A}$, then $M$ is a direct summand of $\operatorname{Hom}_{R}\left(R, R \otimes_{A} M\right)$ by 4.2 (b) so $M \in$ add $\operatorname{Hom}_{R}\left(R, \mathcal{L}_{R}\right)$.

LEMMA 5.3 (a) $\operatorname{add}\left(R \otimes_{A}\right.$ (ind $\left.\left.A \backslash \mathcal{L}_{A}\right)\right)=\operatorname{add}\left(\right.$ ind $\left.R \backslash \mathcal{L}_{R}\right)$. 
(b) $\operatorname{add} \operatorname{Hom}_{R}\left(R\right.$, ind $\left.R \backslash \mathcal{L}_{R}\right)=\operatorname{add}\left(\right.$ ind $\left.A \backslash \mathcal{L}_{A}\right)$.

Proof. We only prove (a) since (b) is similar. Let $M \in \operatorname{ind} A \backslash \mathcal{L}_{A}$ and $R \otimes_{A} M \simeq \bigoplus_{i=1}^{m} X_{i}$ be an indecomposable decomposition in $\bmod R$. By 4.2(c), for any $i$, we have $\operatorname{Hom}_{R}\left(R, X_{i}\right)=$ $\bigoplus_{\sigma \in H_{i}}{ }^{\sigma} M$ for some $H_{i} \subseteq G$. By $5.1,{ }^{\sigma} M \notin \mathcal{L}_{A}$ for any $\sigma$. Hence, by $5.2, X_{i} \notin \mathcal{L}_{R}$ for any $i$. Conversely, assume $X \in \operatorname{ind} R \backslash \mathcal{L}_{R}$. By 4.3, there exists $M \in \operatorname{ind} A$ such that $X$ is a direct summand of $R \otimes_{A} M$. By 5.2, $M \notin \mathcal{L}_{A}$.

We now show that the Ext-injectives in add $\mathcal{L}_{A}$ correspond to those in add $\mathcal{L}_{R}$. For this purpose, we denote by $\mathcal{E}_{1}(A), \mathcal{E}_{2}^{\prime}(A)$ and by $\mathcal{E}_{1}(R), \mathcal{E}_{2}^{\prime}(R)$, respectively, the sets described in Section 3 for the algebras $A$ and $R$.

LEMMA 5.4 (a) $\operatorname{add}\left(R \otimes_{A} \mathcal{E}_{1}(A)\right)=\operatorname{add} \mathcal{E}_{1}(R), \operatorname{add}\left(R \otimes_{A} \mathcal{E}_{2}^{\prime}(A)\right)=\operatorname{add} \mathcal{E}_{2}^{\prime}(R)$.

(b) $\operatorname{add} \operatorname{Hom}_{R}\left(R, \mathcal{E}_{1}(R)\right)=\operatorname{add} \mathcal{E}_{1}(A), \operatorname{add} \operatorname{Hom}_{R}\left(R, \mathcal{E}_{2}^{\prime}(R)\right)=\operatorname{add} \mathcal{E}_{2}^{\prime}(A)$.

Proof. Let $E_{1} \in \mathcal{E}_{1}(A)$ and $X$ be an indecomposable direct summand of $R \otimes_{A} E_{1}$. By 5.2, $X \in \mathcal{L}_{R}$. Now, there exists a path $I \rightsquigarrow E_{1}$ in ind $A$ with $I$ injective. By 4.5 , this path induces a path $I^{\prime} \rightsquigarrow X$ in ind $R$, with $I^{\prime}$ a direct summand of $R \otimes_{A} I$ so that $I^{\prime}$ is an injective $R$-module. Thus $X \in \mathcal{E}_{1}(R)$. Let now $E_{2} \in \mathcal{E}_{2}^{\prime}(A)$ and $X$ be an indecomposable direct summand of $R \otimes_{A} E_{2}$. By 5.2, $X \in \mathcal{L}_{R}$. Moreover, there exists a path $P \rightsquigarrow \tau^{-1} E_{2}$ in ind $A$, with $P \notin \mathcal{L}_{A}$ projective. By $[16,3.8]$, we have that $\tau_{R}^{-1} X$ is a direct summand of $R \otimes_{A}\left(\tau_{A}^{-1} E_{2}\right)$. Applying 4.5 yields a path $P^{\prime} \rightsquigarrow \tau_{R}^{-1} X$ in ind $R$, with $P^{\prime}$ a direct summand of $R \otimes_{A} P$, that is, $P^{\prime}$ is a projective $R$-module. By 5.3, $P^{\prime} \notin \mathcal{L}_{R}$. Then, $X \in \mathcal{E}_{1}(R) \cup \mathcal{E}_{2}^{\prime}(R)$.

One proves in exactly the same way (using 4.7 instead of 4.5 ) that add $\operatorname{Hom}_{R}\left(R, \mathcal{E}_{1}(R)\right) \subseteq$ add $\mathcal{E}_{1}(A)$ and $\operatorname{add} \operatorname{Hom}_{R}\left(R, \mathcal{E}_{2}^{\prime}(R)\right) \subseteq \operatorname{add}\left(\mathcal{E}_{1}(A) \cup \mathcal{E}_{2}^{\prime}(A)\right)$.

Since $\operatorname{add}\left(R \otimes_{A} \mathcal{E}_{1}(A)\right) \subseteq$ add $\mathcal{E}_{1}(R)$ and add $\operatorname{Hom}_{R}\left(R, \mathcal{E}_{1}(R)\right) \subseteq$ add $\mathcal{E}_{1}(A)$, a simple application of $4.2(\mathrm{~b})$ implies that equality holds in each of these cases.

Now, let $E_{2} \in \mathcal{E}_{2}^{\prime}(A)$ and assume that an indecomposable direct summand $X$ of $R \otimes_{A} E_{2}$ lies in $\mathcal{E}_{1}(R)$. Then $\operatorname{Hom}_{R}(R, X) \in \operatorname{add} \mathcal{E}_{1}(A)$. However, $\operatorname{Hom}_{R}(R, X)=\bigoplus_{\sigma \in H}{ }^{\sigma} E_{2}$ for some $H \subseteq G$ so ${ }^{\sigma} E_{2} \in \mathcal{E}_{1}(A)$ for any $\sigma$, hence $E_{2} \in \mathcal{E}_{1}(A)$ a contradiction. This shows that $\operatorname{add}\left(R \otimes_{A}\right.$ add $\left.\mathcal{E}_{2}^{\prime}(A)\right) \subseteq \operatorname{add} \mathcal{E}_{2}^{\prime}(R)$. Similarly, add $\operatorname{Hom}_{R}\left(R, \mathcal{E}_{2}^{\prime}(R)\right) \subseteq \operatorname{add} \mathcal{E}_{2}^{\prime}(A)$. Finally, applying $4.2(\mathrm{~b})$ yields equality in each of these cases.

We recall some definitions. An artin algebra $A$ is left supported if add $\mathcal{L}_{A}$ is contravariantly finite [3]. We know that an algebra $A$ is left supported if and only if add $\mathcal{L}_{A}$ is cogenerated by the direct sum of a complete set of representatives of the isomorphism classes of indecomposable Ext-injectives in add $\mathcal{L}_{A}$ (see [3]). Right supported algebras are defined dually. An artin algebra $A$ is a laura algebra if $\mathcal{L}_{A} \cup \mathcal{R}_{A}$ is cofinite in ind $A$, that is, if ind $A \backslash\left(\mathcal{L}_{A} \cup \mathcal{R}_{A}\right)$ is finite [2]. It is called right glued if the class of all $M \in \operatorname{ind} A$ such that $\operatorname{pd}_{A} M \leq 1$ is cofinite in ind $A$, see [1], or, equivalently, if $\mathcal{L}_{A}$ is cofinite in ind $A$, see [2, 2.2]. Left glued algebras are defined dually. Clearly, left and right glued algebras are laura algebras.

An artin algebra is called weakly shod if the length of any path in ind $A$ from an injective to a projective is bounded. The algebra $A$ is called shod if, for each $M \in$ ind $A$, we have 
$\operatorname{pd}_{A} M \leq 1$ or $\operatorname{id}_{A} M \leq 1$, or, equivalently, if $\mathcal{L}_{A} \cup \mathcal{R}_{A}=$ ind $A$, see [9]. Shod algebras are weakly shod algebras, and weakly shod algebras are laura algebras. Finally, $A$ is quasi-tilted if it is shod and gl.dim. $A \leq 2$, see [12]. Those laura algebras which are not quasi-tilted are called strict. They are left and right supported, see [3, 4.4].

We start the proof of our second main theorem with the following

LEMMA 5.5 Let $A$ and $R$ be as above. Then $A$ is a laura algebra if and only if $R$ is a laura algebra.

Proof. Assume $A$ to be a laura algebra. Then ind $A \backslash\left(\mathcal{L}_{A} \cup \mathcal{R}_{A}\right)$ is finite. Hence the set $\mathcal{X}_{R}$ of all indecomposable $R$-modules in add $\left(R \otimes_{A}\right.$ ind $\left.A \backslash\left(\mathcal{L}_{A} \cup \mathcal{R}_{A}\right)\right)$ is finite. Let $X \notin \mathcal{L}_{R} \cup \mathcal{R}_{R}$ be an indecomposable $R$-module. By 4.3 , there exists $M \in$ ind $A$ such that $M$ is a direct summand of $\operatorname{Hom}_{R}(R, X)$ and $X$ is a direct summand of $R \otimes_{A} M$. By 5.2, $M \notin \mathcal{L}_{A} \cup \mathcal{R}_{A}$. Hence $X \in \mathcal{X}_{R}$ and ind $R \backslash\left(\mathcal{L}_{R} \cup \mathcal{R}_{R}\right) \subseteq \mathcal{X}_{R}$. In particular, ind $R \backslash\left(\mathcal{L}_{R} \cup \mathcal{R}_{R}\right)$ is finite so $R$ is a laura algebra.

Conversely, assume $R$ to be a laura algebra. Hence the set $\mathcal{M}_{A}$ of all indecomposable $A$ modules in add $\operatorname{Hom}_{R}\left(R\right.$, ind $\left.R \backslash\left(\mathcal{L}_{R} \cup \mathcal{R}_{R}\right)\right)$ is finite. Let $M \notin \mathcal{L}_{A} \cup \mathcal{R}_{A}$ be an indecomposable $A$-module. If $R \otimes_{A} M=\bigoplus_{i=1}^{m} X_{i}$ is an indecomposable decomposition in $\bmod R$, then, by 5.3, $X_{i} \notin \mathcal{L}_{R} \cup \mathcal{R}_{R}$ for any $i$. By $4.2(\mathrm{~b}), M$ is a direct summand of $\operatorname{Hom}_{R}\left(R, R \otimes_{A} M\right) \simeq$ $\bigoplus_{i=1}^{m} \operatorname{Hom}_{R}\left(R, X_{i}\right)$. Consequently, $M \in \mathcal{M}_{A}$ and ind $A \backslash\left(\mathcal{L}_{A} \cup \mathcal{R}_{A}\right) \subseteq \mathcal{M}_{A}$. In particular, ind $A \backslash\left(\mathcal{L}_{A} \cup \mathcal{R}_{A}\right)$ is finite, so $A$ is a laura algebra.

The Auslander-Reiten components of a laura algebra have been described in [2]. We notice that, if $A, R$ are as above, and $\Gamma$ is a component of the Auslander-Reiten quiver $\Gamma(\bmod A)$ of $A(\operatorname{or} \Gamma(\bmod R)$ of $R)$, then, in general, the images of the indecomposable modules of $\Gamma$ lie in several components of $\Gamma(\bmod R)(\operatorname{or} \Gamma(\bmod A)$, respectively).

LEMMA 5.6 Let $A$ be an artin algebra, $\Gamma$ be a component of $\Gamma(\bmod A)$ and $\Gamma^{\prime}$ be the unique component of $\Gamma(\bmod R)$ containing an indecomposable direct summand $X$ of $R \otimes_{A} M$, with $M \in \Gamma$. Then:

(a) $\Gamma$ is postprojective if and only if so is $\Gamma^{\prime}$;

(b) $\Gamma$ is preinjective if and only if so is $\Gamma^{\prime}$;

(c) $\Gamma$ is regular if and only if so is $\Gamma^{\prime}$;

(d) $\Gamma$ is non-semiregular if and only if so is $\Gamma^{\prime}$;

(e) $\Gamma$ is semiregular if and only if so is $\Gamma^{\prime}$.

In particular, let $A$ be a strict laura algebra and $\Gamma, \Gamma^{\prime}$ be as above, then $\Gamma$ is the unique faithful non-semiregular component $\Gamma(\bmod A)$ if and only if $\Gamma^{\prime}$ is the unique faithful nonsemiregular component of $\Gamma(\bmod R)$.

Proof. For the proofs of (a), (b) and (c), we refer to [16, 4.3]. We now prove (d). Suppose that $\Gamma$ is non-semiregular, then there exist an indecomposable injective $A$-module $I \in \Gamma$, an 
indecomposable projective $A$-module $P \in \Gamma$, and a walk of irreducible morphisms between indecomposable modules

$$
I=L_{0}-L_{1}-\cdots-L_{s}=M=N_{0}-N_{1}-\cdots-N_{t}=P .
$$

Applying $[16,4.1]$ and induction yields a walk of irreducible morphisms between indecomposable $R$-modules in $\Gamma^{\prime}$

$$
I^{\prime}=Y_{0}-Y_{1}-\cdots-Y_{s}=X=Z_{0}-Z_{1}-\cdots-Z_{t}=P^{\prime}
$$

with $Y_{i}$ a direct summand of $R \otimes_{A} L_{i}$ for any $i$ (thus, $I^{\prime}$ is injective) and $Z_{j}$ a direct summand of $R \otimes_{A} N_{j}$ for any $j$ (thus, $P^{\prime}$ is projective). This shows the sufficiency. The necessity is shown in the same way taking into account that, under the stated hypothesis, $M$ is a direct summand of $\operatorname{Hom}_{R}(R, X)$. Finally, the proof of (e) is also similar.

We are now ready for the proof of our second main theorem.

Proof of Theorem 1.2: The proof of (f) is in [12, II.1.6]. Actually the same proof establishes (e). The proof of $(\mathrm{g})$ in the representation-finite case is in $[16,4.6]$. It carries over to the general case using, for instance, the Liu-Skowroński criterion (see for instance [4, (VIII.5.6) p.342]). The proof of (b) is shown in Lemma 5.5 above. We just show statements (a), (c) and $(\mathrm{d})$.

(a) Assume $A$ to be left supported and denote by $E$ (or $Q$ ) the direct sum of a complete set of representatives of the isomorphism classes of indecomposable Ext-injectives in add $\mathcal{L}_{A}$ (or add $\mathcal{L}_{R}$, respectively). We know that add $\mathcal{L}_{A}$ is the class of $A$-modules cogenerated by $E$. Let $X \in \mathcal{L}_{R}$. Then $\operatorname{Hom}_{R}(R, X) \in \operatorname{add} \mathcal{L}_{A}$ by 5.2 , hence there exist $m>0$ and a monomorphism $\operatorname{Hom}_{R}(R, X) \hookrightarrow E^{(m)}$. Since $X$ is a direct summand of $R \otimes_{A} \operatorname{Hom}_{R}(R, X)$ by 4.2 (b), and $R \otimes_{A}-$ is exact, we deduce a monomorphism

$$
X \hookrightarrow R \otimes_{A} \operatorname{Hom}_{R}(R, X) \hookrightarrow\left(R \otimes_{A} E\right)^{(m)} .
$$

Since $R \otimes_{A} E \in$ add $Q$ by 5.4, $X$ is cogenerated by $Q$. So $R$ is left supported. The converse is proven in the same way.

(c) Assume $A$ to be right glued. Then ind $A \backslash \mathcal{L}_{A}$ is finite. By 5.3 and 4.3 , ind $R \backslash \mathcal{L}_{R}$ is finite. Hence $R$ is right glued. The converse is proven similarly.

(d) Assume $A$ to be weakly shod. If $A$ is quasi-tilted, then so is $R$ (by [12]). We may then assume that $A$ is a strict weakly shod algebra. Then it is a laura algebra and its unique faithful non-semiregular component is directed, by $[2,4.8]$. By $5.5, R$ is also a strict laura algebra, and, by 5.6 and $[16,4.3(\mathrm{a})]$, its unique faithful non-semiregular component is directed. Applying $[2,4.8]$ again, we get indeed that $R$ is strict weakly shod. The converse is proven in the same way.

EXAMPLE Let $k$ be an algebraically closed field of characteristic distinct from 2 (see [16, Section 2]), and $A$ be the radical square zero $k$-algebra given by the quiver 


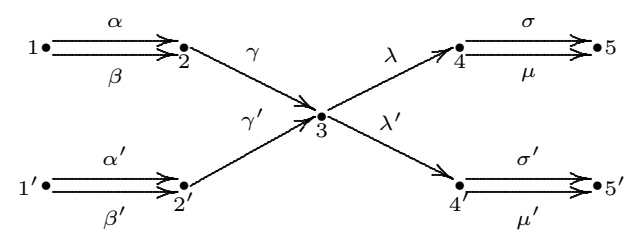

Hence, $A$ is a strict laura algebra. We let the group $\mathbb{Z} / 2 \mathbb{Z}$ act on $A$, where the generator of $\mathbb{Z} / 2 \mathbb{Z}$ fixes the point 3 , permutes the points $x$ and $x^{\prime}$ (for $x \in\{1,2,4,5\}$ ) and the arrows $\xi$ and $\xi^{\prime}$ (for $\xi \in\{\alpha, \beta, \gamma, \lambda, \sigma, \mu\}$ ). Then, by [16, 2.3], $R$ is the radical square zero $k$-algebra given by the quiver

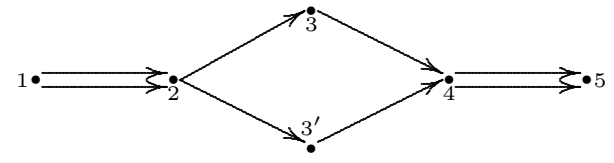

According to our Theorem 1.2, $R$ is also a strict laura algebra. We now draw the unique non-semiregular faithful component of $\Gamma(\bmod A)$.

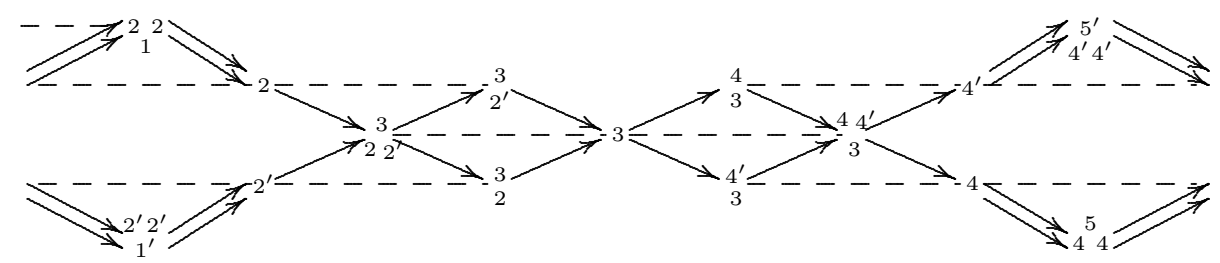

(where indecomposable modules are represented by their Loewy series and horizontal dotted lines describe the Auslander-Reiten translation). The unique non-semiregular faithful component of $\Gamma(\bmod R)$ is

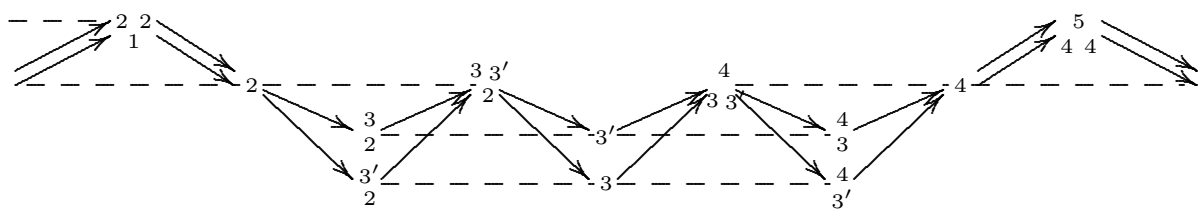

Notice that $\Gamma(\bmod A)$ has two postprojective (or preinjective) components, while $\Gamma(\bmod R)$ has only one.

\section{Skew toupie algebras.}

Throughout this section, all algebras are finite dimensional over an algebraically closed field, thus are bound quiver algebras.

Let $n, l$ be two positive integers. We define the complete bipartite quiver $Q_{l}^{n}$ to have as its only points $n$ sources $a_{1}, \cdots, a_{n}$ and $l$ sinks $b_{1}, \cdots, b_{l}$ and, for each pair $(i, j)$ with $1 \leq i \leq n$, $1 \leq j \leq l$, there is an arrow $a_{i} \rightarrow b_{j}$ and these are the only arrows of $Q_{l}^{n}$. A skew toupie quiver $Q$ is defined as follows: it consists of a complete bipartite quiver $Q_{l}^{n}$, its opposite quiver $\left(Q_{l}^{n}\right)^{o p}$ as well as $l$ disjoint paths $w_{1}, \cdots, w_{l}$ from the sinks of $Q_{l}^{n}$ to the sources of 
$\left(Q_{l}^{n}\right)^{o p}$. Thus, a skew toupie quiver has the form:

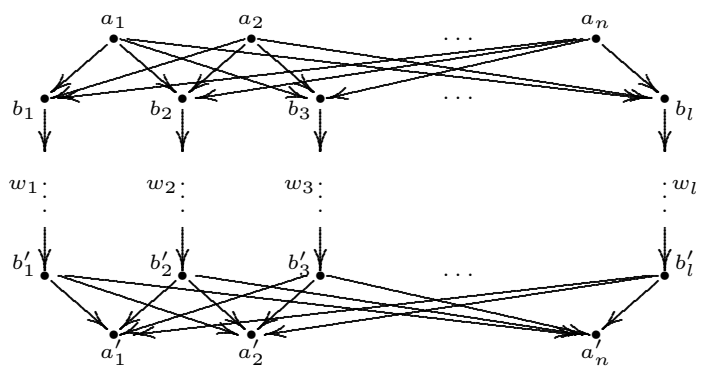

An algebra $R=k Q / I$ is called a skew toupie algebra if its quiver $Q$ is a skew toupie quiver. Our objective in this section in to exhibit a family of skew toupie algebras which are laura, and even weakly shod.

If, above, $Q$ has exactly one source and one sink (that is, $n=1$ ), then $Q$ is a toupie quiver, and $R=k Q / I$ is a toupie algebra, as defined and studied in [7].

PROPOSITION 6.1 Let $R=k Q / I$ be a skew toupie algebra with $m=n l$ branches, $k$ an algebraically closed field and $n$ invertible in $k$. Then $R$ is a weakly shod algebra provided:

(1) The ideal I is generated by all possible commutativity relations. In this case, $R$ is tilted.

(2) The ideal I is monomial, and generated by at least one subpath of each of the $w_{i}$. In this case, $R$ is tilted if and only if each path $w_{i}$ is bound by exactly one relation.

(3) The ideal $I$ is generated by the sums of all paths from each source to each sink, and moreover $n l \in\{2,3\}$ or the length of each $w_{i}$ does not exceed one. In this case, $R$ is canonical if $l=3$, and tilted otherwise.

Proof. We consider the toupie quiver $Q^{\prime}$ with $m=n l$ branches

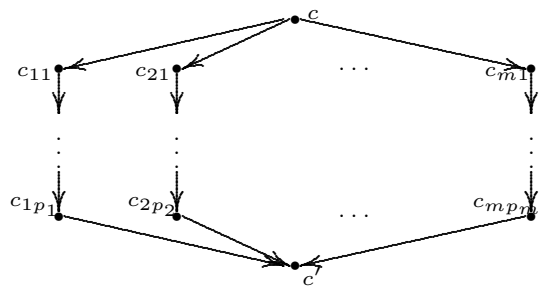

For each $i$ with $1 \leq i \leq m$, we set $i^{\prime}=i+l$ if $i+l \leq m$ and $i^{\prime}=i+l-m$ if $i+l>m$. We suppose that, for each $i$ with $1 \leq i \leq m$, we have $p_{i}=p_{i^{\prime}}$ and, if $i \leq l$, we set $p_{i}=\ell\left(w_{i}\right)+1$, where $\ell\left(w_{i}\right)$ denotes the length of the path $w_{i}$. We finally denote by $\gamma_{i}$ the path $c_{i 1} \rightarrow c_{i 2} \rightarrow \cdots \rightarrow c_{i p_{i}}$, for $1 \leq i \leq m$.

We define on $Q^{\prime}$ an action of the cyclic group $\mathbb{Z} / n \mathbb{Z}$ as follows: let $\sigma$ denote a generator of $\mathbb{Z} / n \mathbb{Z}$, then set $\sigma(c)=c, \sigma\left(c^{\prime}\right)=c^{\prime}$ and, for each pair $(i, j)$, with $1 \leq i \leq m$ and $1 \leq j \leq p_{i}$, $\sigma\left(c_{i j}\right)=c_{i^{\prime} j}$ where $i^{\prime}$ is as above. We let $\sigma$ have the induced action on the arrows. This defines indeed an action on $Q^{\prime}$ (and hence on the path algebra $k Q^{\prime}$ ) because of our assumption on the $p_{i}$.

Let $A=k Q^{\prime} / I^{\prime}$, where $I^{\prime}$ is an admissible ideal of one of the following forms: 
1. $I^{\prime}$ is generated by all possible commutativity relations;

2. $I^{\prime}$ is a monomial ideal generated by at least one subpath of each of the $\gamma_{i}$. Moreover, for each $i$ such that $1 \leq i \leq m$, the path $\gamma_{i}$ is isomorphic to $\gamma_{i^{\prime}}$, as full convex subcategories of $A$ (again, $i^{\prime}$ is as above);

3. $I^{\prime}$ is generated by the sum of all paths from $c$ to $c^{\prime}$ and moreover $m \in\{2,3\}$ or $p_{i} \leq 2$ for all $i$.

Clearly, the action of $\mathbb{Z} / n \mathbb{Z}$ on $k Q^{\prime}$ leaves invariant the ideal $I^{\prime}$. Thus, by [16, 2.1], $\mathbb{Z} / n \mathbb{Z}$ acts on $A$. Now, it follows from the main result of [7] that, in each of these cases, $A$ is a weakly shod algebra. Furthermore, in the case (1) it is always tilted, while, in the case (2), it is tilted if and only if each path $\gamma_{i}$ is bound by exactly one relation, and, finally, in the case (3), it is canonical if and only if $m=3$ and $l>1$ (thus $l=3$ ) and tilted in all the other cases. By $[16,2.3]$, we get $A[\mathbb{Z} / n \mathbb{Z}]^{b}=R$, as given in the statement of our proposition. The assertion now follows from Theorem 1.2.

EXAMPLE Let $k$ be an algebraically closed field of characteristic distinct from 3 , and $A$ be given by the toupie quiver

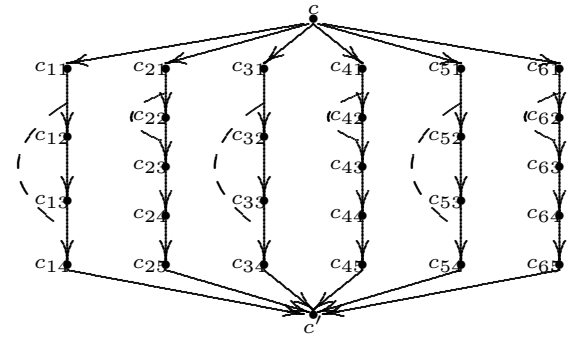

bound by the relations denoted by the shown dotted lines. We define an action of $\mathbb{Z} / 3 \mathbb{Z}=<$ $\sigma>$ as follows: $\sigma(c)=c, \sigma\left(c^{\prime}\right)=c^{\prime}, \sigma\left(c_{1 i}\right)=c_{3 i}, \sigma\left(c_{3 i}\right)=c_{5 i}$ for $1 \leq i \leq 4, \sigma\left(c_{2 j}\right)=c_{4 j}$, $\sigma\left(c_{4 j}\right)=c_{6 j}$ for $1 \leq j \leq 5$. In this case, $R$ is given by the quiver

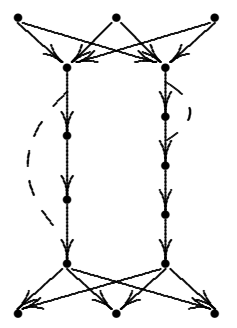

bound by the relations denoted by the shown dotted lines. According to $6.1, R$ is tilted.

ACKNOWLEDGEMENTS. The first author gratefully acknowledges partial support from the NSERC of Canada. This paper was written while the second author was a CRMISM postdoctoral fellow at the universities of Sherbrooke and Bishop's. This paper was started while the third author was visiting the University of Sherbrooke in Québec. She 
acknowledges support from the NSERC of Canada, and would like to express her gratitude to Ibrahim and Marcelo for their warm hospitality.

\section{References}

[1] Assem, I.; Coelho, F. U. Glueings of tilted algebras. J. Pure Appl. Algebra 96 (1994), no. $3,225-243$.

[2] Assem, I.; Coelho, F. U. Two-sided gluings of tilted algebras. J. Algebra 269 (2003), no. $2,456-479$.

[3] Assem, I.; Coelho, F. U.; Trepode, S. The left and the right parts of a module category. J. Algebra 281 (2004), 518-534.

[4] Assem, I.; Simson, D., Skowroński, A., Elements of the representation theory of associative algebras, Vol. 1, London Math. Soc. Student Texts 65 (2006) Cambridge Univ. Press.

[5] Auslander, M.; Reiten, I.; Smalø, S. Representation theory of Artin algebras. Cambridge Studies in Advanced Mathematics, 36. Cambridge University Press, Cambridge, 1997. xiv $+425 \mathrm{pp}$.

[6] Auslander, M.; Smalø, S. Almost split sequences in subcategories. J. Algebra 69 (1981), no. $2,426-454$.

[7] Castonguay, D.; Huard, F.; Lanzilotta, M. Toupie algebras. In preparation.

[8] Cibils, C.; Marcos, E. Skew category, Galois coverings and smash product of a $k$ category. Proc. Amer. Math. Soc. 134 (2006), no. 1, 39-50.

[9] Coelho, F. U.; Lanzilotta, M. Algebras with small homological dimensions. Manuscripta Math. 100 (1999), no. 1, 1-11.

[10] Coelho, F. U.; Lanzilotta, M. Weakly shod algebras. J. Algebra 265 (2003), no. 1, 379-403.

[11] Funes, O.; Redondo, M. J. Skew group algebras of simply connected algebras. Ann. Sci. Math. Québec 26 (2002), no. 2, 171-180.

[12] Happel, D.; Reiten, I.; Smalø, S. Tilting in abelian categories and quasitilted algebras. Mem. Amer. Math. Soc. 120 (1996), no. 575, viii+ 88 pp.

[13] de la Peña, J. A. Twisted algebras and coverings. Preprint UNAM, 1983.

[14] de la Peña, J. A. Automorphisms and twisted algebras. Preprint UNAM, 1983. 
[15] de la Peña, J. A. Automorfismos, álgebras torcidas y cubiertas, PhD Thesis, UNAM, 1983.

[16] Reiten, I. ; Riedtmann, Ch. Skew group algebras in the representation theory of Artin algebras. J. Algebra 92 (1985), no. 1, 224-282. 